\title{
QUELQUES DONNÉES RELATIVES A L'EXPLOITATION DES RÉSEAUX D'IRRIGATION EN ITALIE DU NORD
}

\author{
SOME DATA RELATIVE TO THE EXPLOITATION OF IRRIGATION \\ NETWORKS IN NORTHERN ITALY
}

par R. RÉ

(English synopsis p. 775)

\section{LES PROBLEMMES \\ DE LA DISTRIBUTION DE L'EAU}

Les opérations de distribution, en beaucoup de domaines, ont toujours posé des problèmes d'organisation parfois délicats à résoudre, plus ou moins facilités ou compliqués suivant les positions relatives du distributeur et des réceptionnaires.

Distribuez des cartes d'alimentation à un peuple, et vous serez effrayé par le nombre, mais vous vous consolerez en pensant que les intéressés viendront chercher eux-mêmes leurs tickets. Ravitaillez des armées en campagne et votre souci grandira, car il vous faudra jouer à cache-cache avec vos unités. Groupez en syndicat les agriculteurs d'une contrée et essayez de leur assurer le service de l'eau d'irrigation et vous rencontrerez aussi de grandes difficultés dont nous allons succinctement rappeler les principales.

La première des difficultés tient à la nature du produit distribué. Le transport de l'eau comparé à celui des corps solides par exemple, pose à lui seul une multitude de problèmes. Heureusement pour nous, l'hydraulique des canaux et les mille et un appareils ingénieux inventés par l'homme pour asservir cet élément essentiellement capricieux qu'est l'eau, sont là pour nous venir en aide.

Une deuxième difficulté réside dans le fait que de grandes distances séparent les têtes de distributions des ultimes points à desservir. La multitude de ces derniers, au nombre de plusieurs dizaines de milliers souvent, les variations des attributions et des disponibilités suivant les époques, ne facilitent pas la tâche.

Indépendamment des facteurs que nous venons d'évoquer, il ne faut pas oublier que ce travail de distribution doit être effectué le plus économiquement possible et entraîner un ensemb!e de dépenses à l'échelle de la valour intrinsèque de l'eau, c'est-à-dire quelques dizaines de centimes au $\mathrm{m}^{3}$ pour la période actuelle.

Enfin, le projet de réseau d'irrigation ne conduit pas seulement à un ensemble d'ouvrages à réaliser moyennant certaines dépenses de premier établissement, il conduit aussi à un mode de fonctionnement et à une somme de travaux d'entretien dont le budget annuel est à mettre en regard avec les annuités d'amortissement du réseau lui-même.

Le système de transport des eaux étant fixé, comment l'utilise-t-on, comment le personnel d'exploitation est-il employé, par quelles opérations chaque usager reçoit-il ses attributions d'eau? Comment l'exploitation pratique des réseaux d'irrigation s'accommode-t-elle des difficultés que nous venons de signaler? Tel est le sujet de notre article. Laissant délibérément de côté les modes de réalisation de la distribution uniquement mentionnés pour une bonne compréhension de l'exposé, nous nous proposons d'analyser le fonctionnement des organismes hábilités à fournir de l'eau d'irrigation que nous avons pu visiter.

C'est en Italie du Nord, pays où l'irrigation se pratique depuis de nombreux siècles et continue à se développer à un rythme de plus en plus rapide, que nous avons commencé notre enquête. Nous allons exposer les grandes lignes de marche de trois des réseaux parmi les plus importants de la rive gauche du Pô.

Auparavant, il nous a paru bon de rappeler quelques définitions, dans le but d'éviter toute confusion dans les termes employés. Il convient surtout de se pénétrer de la réelle signification 
de la main d'eau du débit fictif continu et du module d'irrigation.

\section{Main d'eau.}

C'est le débit réellement appliqué au terrain; suivant les pays, les terrains, les cultures, la manière d'arroser, ce débit varie dans de très larges proportions, depuis quelques litres jusqu'à 300 à la seconde.

Pour réaliser un arrosage, l'irrigant conduit la main d'eau dans ses plantations pendant un certain temps, le temps d'arrosage. Après quoi cette main d'eau est utilisée par un autre usager.

\section{Débit fictif continu.}

Considérons une parcelle donnée, un intervalle de temps assez long, semaine, mois ou même saison, le nombre et la durée des arrosages pratiqués, et la valeur de la main d'eau utilisée. Pendant le laps de temps considéré, la parcelle reçoit un volume d'eau bien déterminé, à partir duquel il est àisé de calculer le volume qu'aurait reçu chaque hectare de la parcelle, pendant chaque seconde, si l'eau d'arrosage avait été appliquée sans discontinuité. C'est ce dernier nombre exprimé en litres/seconde/hectare que nous appellerons débit fictif continu.

\section{Module d'irrigation.}

Si, au lieu de considérer une parcelle donnée. nous considérons l'ensemble du réseau, nous définirons de la même manière un volume d'eau total attribué et un débit fictif continu. Si le réseau est suffisamment grand, et c'est en général le cas, ce débit n'est pas fictif, mais bien réel. Si $N$ est le nombre d'hectares du réseau, c'est la Nième partie du débit qui est réellement introduit en tête du réseau; nous l'appellerons alors module d'irrigation.

\section{LE RÉGIME D'EXPLOITATION DES RÉSEAUX D'IRRICATION DE L'ITALIE SEPTENTRIONALE}

Le bassin du Pô est l'un de ceux au monde où l'aménagement à l'irrigation est proportionnellement le plus développé.

A la sortie des montagnes, aussitôt qu'elles débouchent dans la plaine, les rivières sont immédiatement mises à contribution et une très grosse part des terres de la plaine reçoivent de l'eau. Aujourd'hui c'est une région magnifique non par la grâce de la nature, mais à la suite d'un labeur persévérant commencé il y a dix siècles. Au nord du Pô, les alluvions sont essentiellement des sables et des graviers, assez fins au voisinage du fleuve, de plus en plus grossiers en se rapprochant des montagnes. Des générations l'une après l'autre ont travaillé à rassembler les gros éléments à l'écart, et. sur les matériaux plus fins restant, l'eau et l'argent aidant, le sol a été presque artificiellement créé ; ce sol constitue aujourd'hui la richesse de cette région

Totalement différentes sont les terres de la rive droite du Pô. Au lieu des alluvions sableuses et gravelleuses, on trouve de l'argile formée par les matériaux arrachés aux Apennins et lentement colmatés. Mais là aussi, après beaucoup d'efforts, l'homme a réussi à instaurer une économie agricole des plus productrices.

Les réseaux dont nous parlerons se situent au nord du Pô. Les irrigations qu'on y rencontre s'effectuent donc sur des terrains excessivement perméables

Cette grosse perméabilité du terrain s'allie parfois à l'impénétrabilité de certaines cultures telles que les herbages, pour gêner considérablement l'avancement de l'eau sur le terrain et nécessiter des mains d'eau considérables atteignant couramment 250 et même $300 \mathrm{l} . / \mathrm{sec}$.

L'extension des réseaux d'irrigation en Italie septentrionale a rencontré de grosses difficultés dues à la très grande ancienneté des irrigations. En effet, les premiers essais d'importance furent tentés à la suite de la paix de Costanza (1183), par laquelle les communes lombardes retrouvèrent la liberté et obtinrent la reconnaissance de la pleine juridiction de leurs fleuves.

Depuis, la structure des Etats a maintes et maintes fois changé au cours de l'histoire, mais les irrigants se sont toujours servis de l'eau. Leur nombre s'est accru. Mais souvent le privilège de premier utilisant subsistait et la création d'irrigations nouvelles, toujours au voisinage d'anciennes, était considérablement gênée par cet état de fait.

L'acte le plus significatif de la législation italienne en matière d'utilisation des eaux est la loi de 1916 qui mit le frein aux spéculations abusives dont l'eau était l'enjeu, en attribuant la propriété de l'eau à l'Etat et à l'Etat seul. Les usagers ne peuvent être que concessionnaires. Mais les concessions ont revêtu des caractères différents suivant l'étendue du droit dont jouissait le premier propriétaire, si bien que tout n'est pas encore régulièrement ordonné et tous les 
utilisateurs ne sont pas encore servis de la même manière.

En gros, il existe deux sortes de réseaux :

- les réseaux d'Etat, où les canaux sont exécutés par l'Etat et gérés par lui ;

- les réseaux syndicaux, où les canaux sont construits par le Syndicat avec subvention d'Etat. L'eau est concédée par l'Etat au Syndicat à un taux faible, le Syndicat ayant la charge de la gérance.

Le canal Cavour, par exemple, est un canal d'Etat. Les canaux qui en partent sont syndicaux.

La carte schématique de l'Italie du Nord figurée à la planche I indique l'emplacement des trois réseaux dont nous allons décrire le mode d'exploitation. Comme nous l'avons déjà signalé, ils s'étendent au nord du Pô et utilisent les eaux des rivières descendant des Alpes.

\section{SYNDICAT VILLORESI}

Le réseau Villoresi, du nom de l'Ingénieur qui le projeta et le défendit, intéresse 60.000 hectares environ situés sur la rive gauche du Tessin, au nord et au voisinage de la ville de Milan (planche 2).

Le canal Villoresi prend ses eaux dans le Tessin et déroule vers l'est ses 60 kilomètres de développement, dominant ainsi une bande de terrain dont la largeur varie entre 2 et $15 \mathrm{~km}$.

Remarquons que cet important réseau dessert une région où la pluviométrie annuelle est de l'ordre de $1.000 \mathrm{~mm}$., dont environ $250 \mathrm{~mm}$ pour le trimestre d'été, juin, juillet, août.

La population est dense : 554 habitants au $\mathrm{km}^{2}$, parmi lesquels 65 ont une occupation purement agricole, et 50 une occupation agricole secondaire.

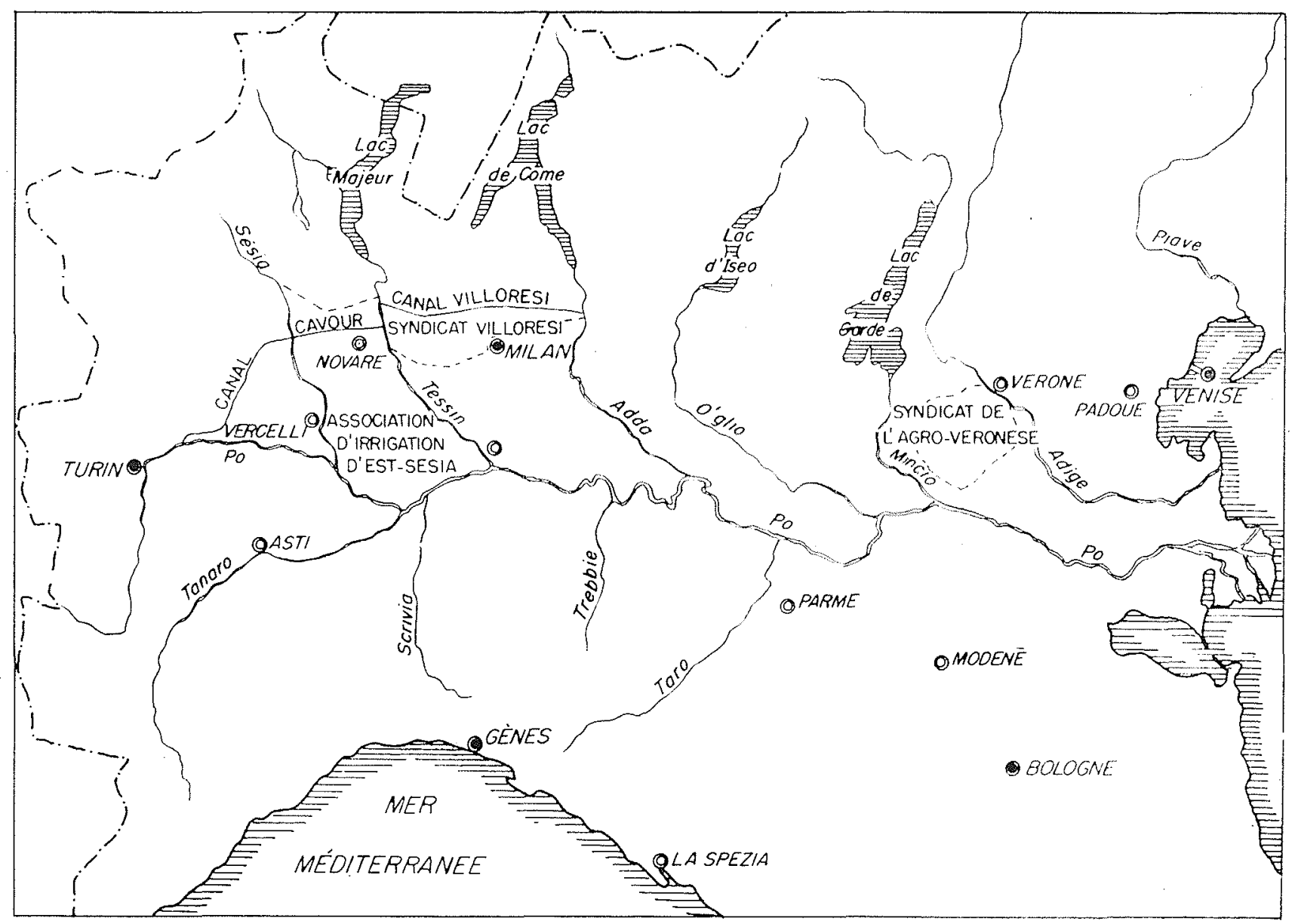

Planche $N^{\circ} 1$

L'Italie du Nord 


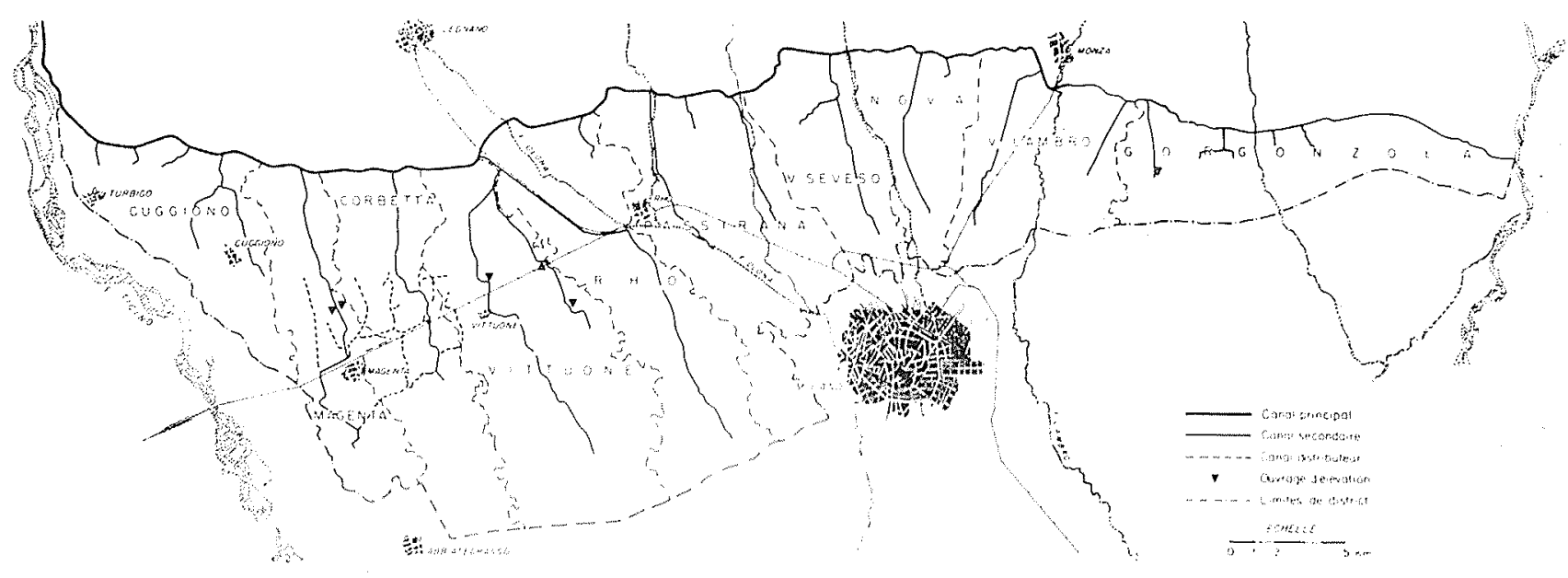

Planche $N=2$

Le Réseau Villoresi

La propriété est très morcelée.

$45 \%$ du terrain sont occupés par des propriétés de moins de 0,5 hectares.

$22 \%$ par des propriétés comprises entre 0,5 et 1 hectare.

$18 \%$ par des propriétés comprises entre 1 et 2 hectares.

$3 \%$ seulement par des propriétés supérieures à 10 hectares.

La superficie moyenne de la parcelle cadastrale n'est que de $670 \mathrm{~m}^{2}$.

Pour une surface effectivement irriguée de 33.000 hectares, le nombre des propriétaires atteint 16.000, celui des usagers 22.000 .

La répartition des cultures est sensiblement la suivante :

$26 \%$ de blé

$25 \%$ de maïs,

$19 \%$ de prairies artificielles,

$11 \%$ de prés.

Le reste en cultures diverses.

L'organisation du service d'exploitation est la suivante :

A Milan se trouve le siège central du réseau (consorzio).

Ce bureau central se compose:

d'un Directeur général (en l'occurrence M. SCIAVETTI),

3 ingénieurs,

4 géomètres-dessinateurs.

1 chef comptable,

2 comptables,

5 employés.
Le réseau est divisé en 10 districts (distretti), chacun d'eux desservi par un canal secondaire. Ces districts sont groupés par 3 ou 4 pour constituer une zone à la tête de laquelle se trouve un bureau de zone, composé de:

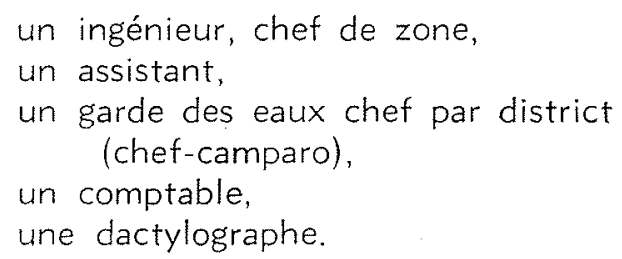

Chaque district est divisé en "comizi 》 (100 à 300 ha). C'est la superficie irriguée par un tertiaire ou deux, suivant leur importance. Chaque « comizio» groupe 50 à 300 usagers. A chaque "comizio" est affecté un camparo (textuellement c'est un manœuvre; bien qu'il effectue la distribution et le gardiennage, on ne l'appelle pas garde des eaux, car il n'est employé que pendant la saison d'irrigation). Ce camparo surveille deux mains d'eau, c'est-à-dire environ $5001 . / \mathrm{sec}$.

\section{Principe de la distribution.}

Dans chaque tertiaire coule une main d'eau (en principe de $2501 . / \mathrm{sec}$.) qui est attribuée une fois par semaine intégralement et par rotation à chaque dépendant du tertiaire.

L'ordre des arrosages à l'intérieur de chaque tour est défini une fois pour toutes au début de la saison. Si les débits disponibles en tête du réseau varient au cours de la saison, ce sont les mains d'eau qui subissent des variations proportionnelles. 

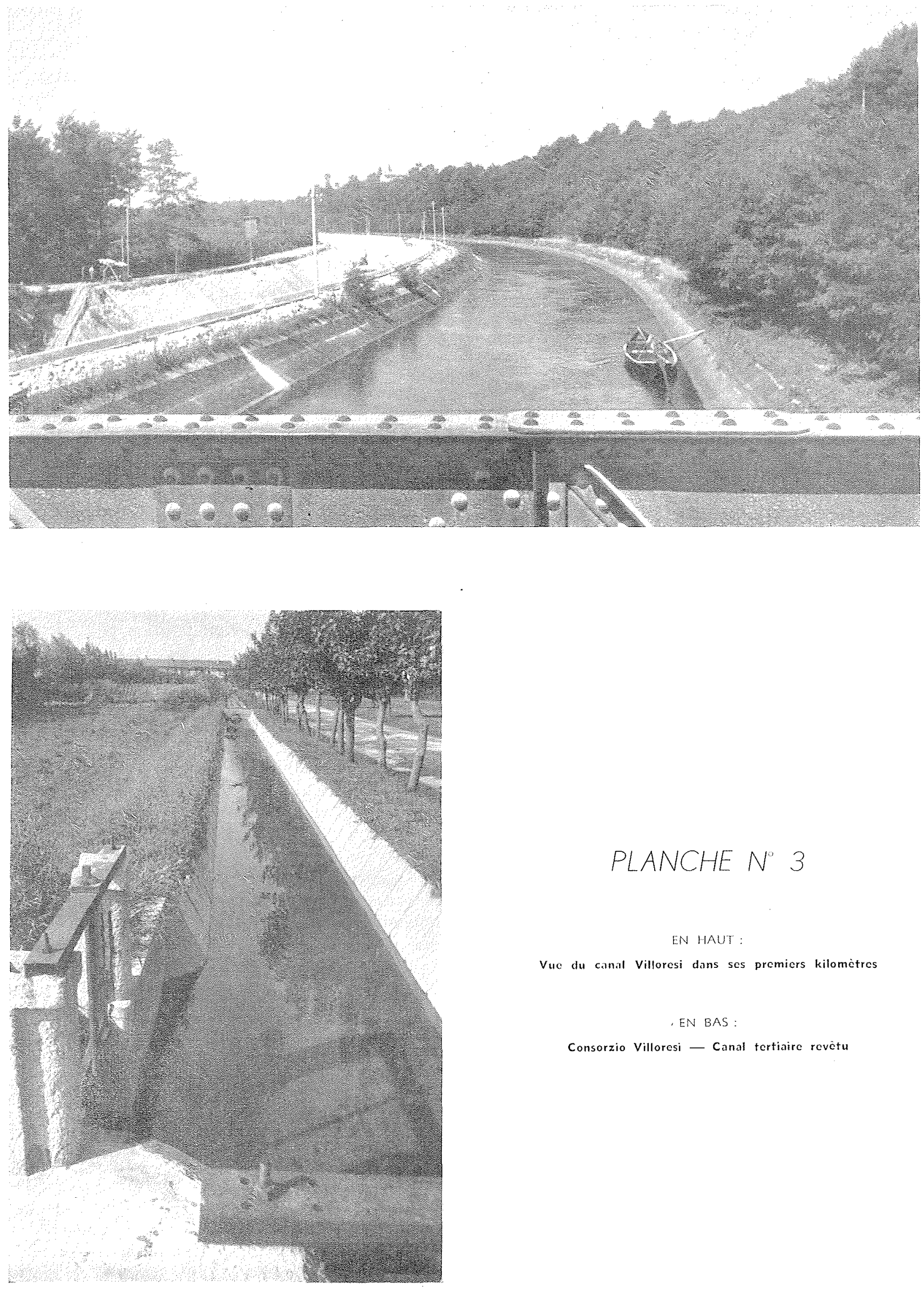

PLANCHE N 3

EN HAUT:

Vue du canal Villoresi dans ses premiers kilomètres

- EN BAS:

Consorzio Villoresi - Canal tertiaire revétu 
Avant le commencement de la saison, les usagers remplissent une demande imprimée dont on trouvera une reproduction sur la planche No 4 .

Les irrigants déclarent les superficies de leurs différentes cultures, l'eau qui leur a été accordée au cours de la saison précédente et celle qu'ils demandent pour la saison à venir.

L'eau attribuée peut l'être de deux manières:

\section{a) Par rotation.}

C'est-à-dire qu'une fois par semaine ils disposent de la main d'eau qui dessert le comizio dont ils dépendent.

On remarquera que la demande doit se faire en litres, chose aisée, car l'imprimé porte l'indication : à chaque litre d'eau syndicale correspondent $x$ heures d'arrosage.

Exemple:

La main d'eau est de $2501 . / \mathrm{sec}$.

La semaine comprend $24 \times 7=168$ heures.

Une heure d'arrosage avec 2501 ./ sec. correspond à un débit fictif continu de :

$$
\frac{250}{168}=1.491 . / \mathrm{sec} \text {. }
$$

Inversement, un litre/seconde accordé correspond à un arrosage de :

$$
\frac{1 \text { heure }}{1,49}=40 \text { minutes. }
$$

Remarquons que l'imprimé distingue les eaux communes des eaux syndicales.

Les eaux communes ne sont pas du ressort du Syndicat. ( $\mathrm{Ce}$ sont des sources particulières ou des eaux fournies par de petits cours d'eau indépendants du canal principal du réseau). Ces eaux communes sont tout de même sujettes à taxe, mais peu élevée.

\section{b) D'une manière continue.}

Ceci a lieu pour les rizières où il est nécessaire que l'eau arrive sans discontinuité, mais avec évidemment des débits plus faibles.

\section{Exemple:}

A un champ de riz de 5 hectares, il sera affecté en permanence $101 . / \mathrm{sec}$.

Ces demandes sont rassemblées au siège central qui les examine.

Des précisions sont demandées à l'usager si sa demande diffère de l'attribution de la saison dernière.

Puis on établit les tours d'eau.

Transformée en heures, la somme des demandes d'un comizio doit faire 168 heures. On modifie légèrement chaque demande pour que la somme fasse exactement 168 ; on ne s'écarte jamais beaucoup, puisqu'on a vérifié que chaque demande est sensiblement la même que celle de l'année précédente

On fixe alors l'horaire d'arrosage de chacun.

Le premier usager commencera le lundi à 0 heure, et pendant 3 h. 45 par exemple, le suivant de 3 h. 45 à 7 heures, etc..., et le lundi suivant le premier usager commencera à arroser à la même heure.

Cet horaire, établi une fois au début de la saison, reste valable toute la saison.

Ces bulletins, rassemblés par les bureaux de la zone qui les examinent (en particulier, ils relèvent les demandes exagérées et demandent des explications), sont ensuite envoyés au bureau central qui établit les horaires.

La deuxième opération consiste à établir le devis des dépenses:

- pour les mancuvres de vannes et le gardiennage ;

- pour l'entretien du réseau.

Ce devis étant fait, on détermine le prix de revient du litre/seconde et il est alors facile d'établir les bulletins de paiement de chaque usager.

Ce bulletin est composé de trois parties détachables (planche $\left.N^{0} 5\right)$ :

- La partie de gauche est renvoyée par l'usa. ger au Syndicat comme accusé de réception de l'avis de paiement.

- La partie centrale, lorsque l'usager s'est acquitté de la contribution auprès d'une des banques accréditées par le Syndicat, est conservée par l'usager à titre de quittance.

- La troisième partie, celle de droite, est adressée par la banque, où a eu lieu le paiement, au Syndicat, qui se rend ainsi compte des retards de paiement.

La dernière tâche du bureau central est d'établir les devis pour les nouveaux travaux: construction de canaux nouveaux, revêtements de canaux qui n'ont jamais été revêtus, creusement 


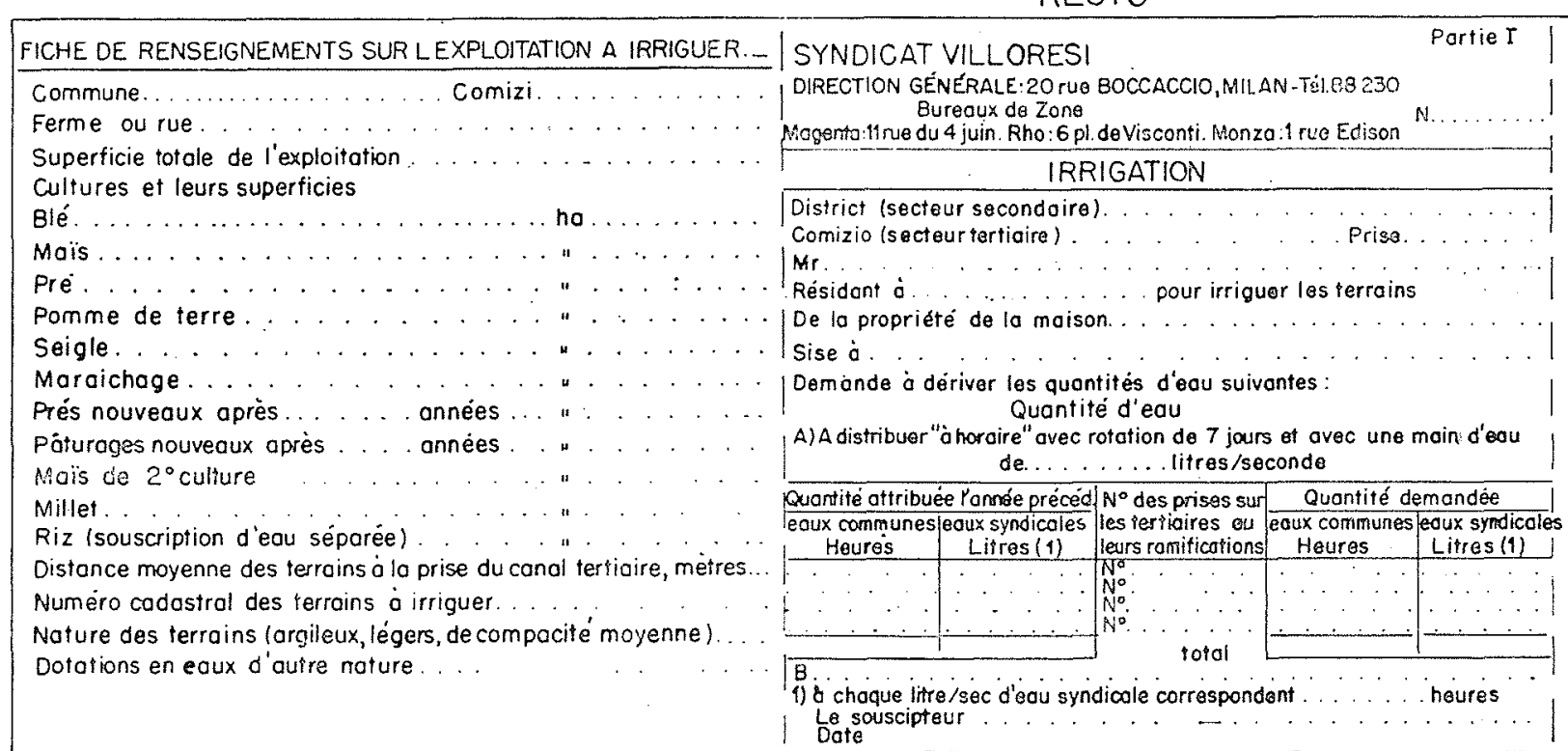

VERSO

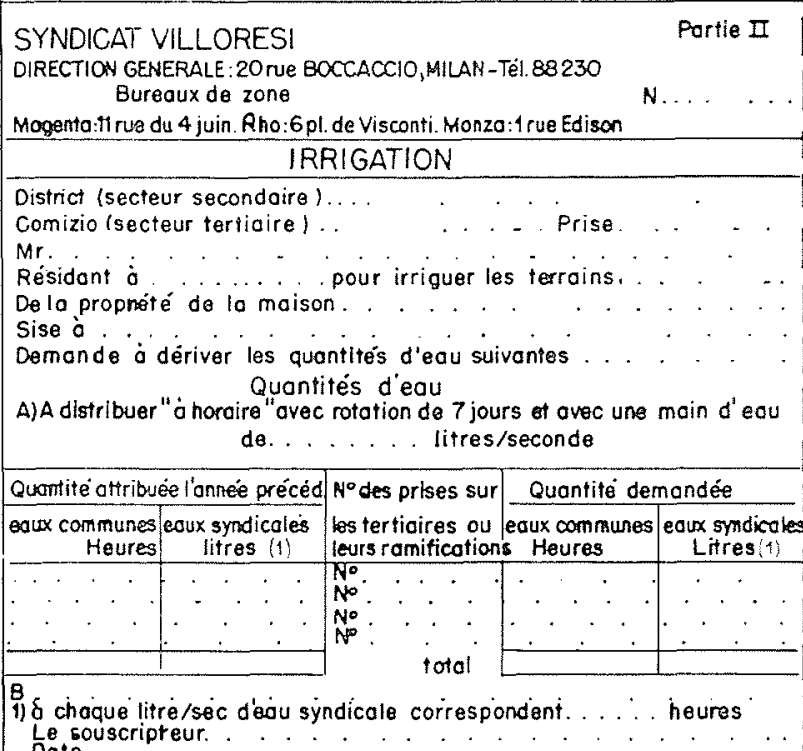
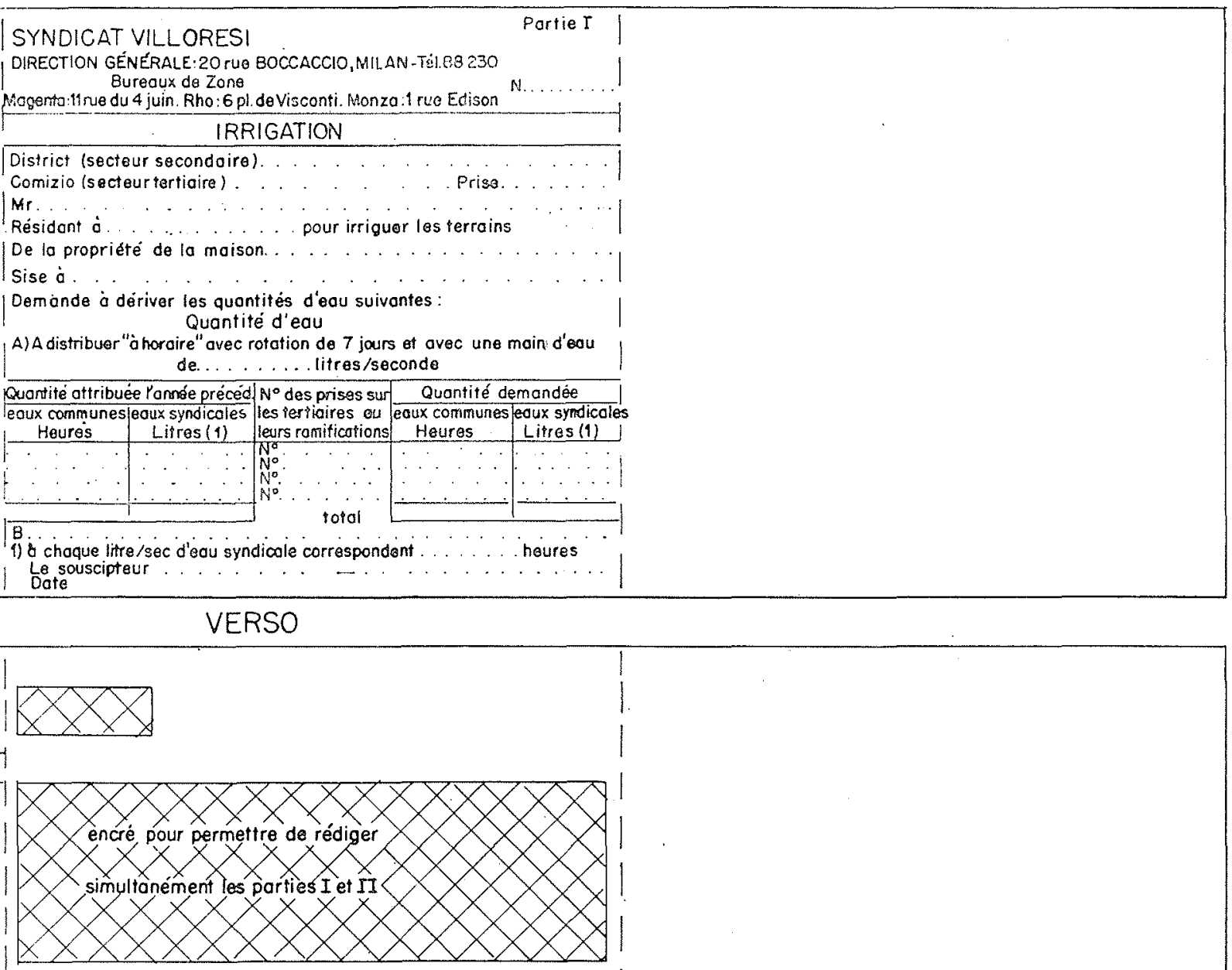

|

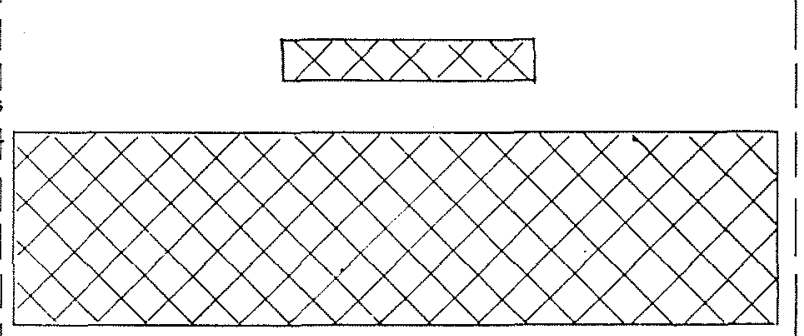


de nouveaux canaux de drainage. Tous les autres petits travaux entrent dans le chapitre entretien.

\section{ROLE DES DIFFÉRENTS BUREAUX DU SYNDICAT}

\section{Bureau central.}

Nous venons donc de définir les tâches du bureau central que nous résumons ainsi :

l"Rassemblement des souscriptions et établissement des horaires;

$2^{\circ}$ Préparation du devis des dépenses de gérance et d'entretien ;

30 Emission des bulletins de paiement;

4" Préparation des devis de travaux neufs

\section{Bureau de zone.}

- Il établit le compte des dépenses de la zone pour chaque quinzaine.

- Il présente les projets de travaux neufs.

- L'assistant surveille les chefs-campari et les campari. II dispose d'une motocyclette et surveille les travaux en cours.

\section{District.}

Le chef-campari surveille les campari.

Quant aux campari, ce sont des ouvriers embauchés seulement à la saison. En été, ils effectuent les manœuvres de vannes. Certains sont embauchés l'hiver pour le curage des canaux.

Chef-campari et campari sont munis d'une bicyclette.

\section{Prix de l'eau.}

Il y a deux contributions:

a) la contribution de consommation, qui est proportionnelle au débit souscrit en début de saison. C'est celle qui couvre les frais de gérance du Syndicat. Elle est payée par le consommateur effectif de l'eau ;

b) la contribution syndicale proportionnelle à la surface, qui couvre les dépenses de travaux neufs et qui est en somme attachée au terrain. Elle est payée par les propriétaires.

Avant guerre, les contributions étaient les suivantes:

pour a) : 50 à 60 lires au $1 . / \mathrm{sec}$.

"b) : 60 lires à l'hectare.

Aujourd'hui :

pour a) : 3.000 lires au I./sec.

$»$ b) : 1.200 lires à l'hectare.
Le chiffre d'affaires de la saison 1948 est :

pour a) : 160 millions.

» b) : 80 millions, dont la moitié est couverte par un emprunt.

Les banques où s'effectuent les paiements assurent la trésorerie du Syndicat.

\section{CONSORZIO D'IRRIGAZIONE DELL'AGRO VERONESE}

La grande salle de réunion de ce Syndicat, à Vérone, est ornée d'une immense carte du réseau, sur laquelle nous avons relevé les indications suivantes:

Superficie du réseau ... 26.362 hr. Superficie nette irriguée 23.947 ha. Superficie à irriguer ... 2.415 ha. Débit concédé au réseau $\quad 27 \mathrm{~m}^{3} / \mathrm{sec}$. Besoins d'eau à l'hectare 0,8 à 1,41 . $/ \mathrm{sec}$ Longueur des canaux d'irrigation .......6 604.000 mètres

Longueur des canaux

de colature ....... 50.000 mètres

Les cultures principales sont identiques à celles du consorzio Villoresi, à savoir : blé, prairies et maïs.

Le réseau prend ses eaux à partir de l'Adige. Plusieurs syndicats sont alimentés par la même tête-morte, et ce n'est qu'à Vérone que le Syndicat de l'Agro Veronese prend en charge les eaux qui lui reviennent.

Le personnel de gérance comprend: Un Directeur général ( $M$. ABATTI), Un adjoint technique avec:

1 dessinateur

1 employé

Un Secrétaire administratif avec:

$$
1 \text { dactylo }
$$$$
1 \text { secrétaire }
$$

Un assistant qui est le chef du gardiennage avec:

3 chefs gardiens

17 gardiens

II est affecté un gardien par secondaire. Ce gardien surveille 50 à $60 \mathrm{~km}$. de canaux. II est muni d'une bicyclette et effectue deux tourriées par jour (le long du secondaire), même s'il n'y a pas de mancuvres à effectuer.

Remarquons que ce gardien a le droit de dresser contravention en matière d'infractions au règlement de distribution des eaux. 


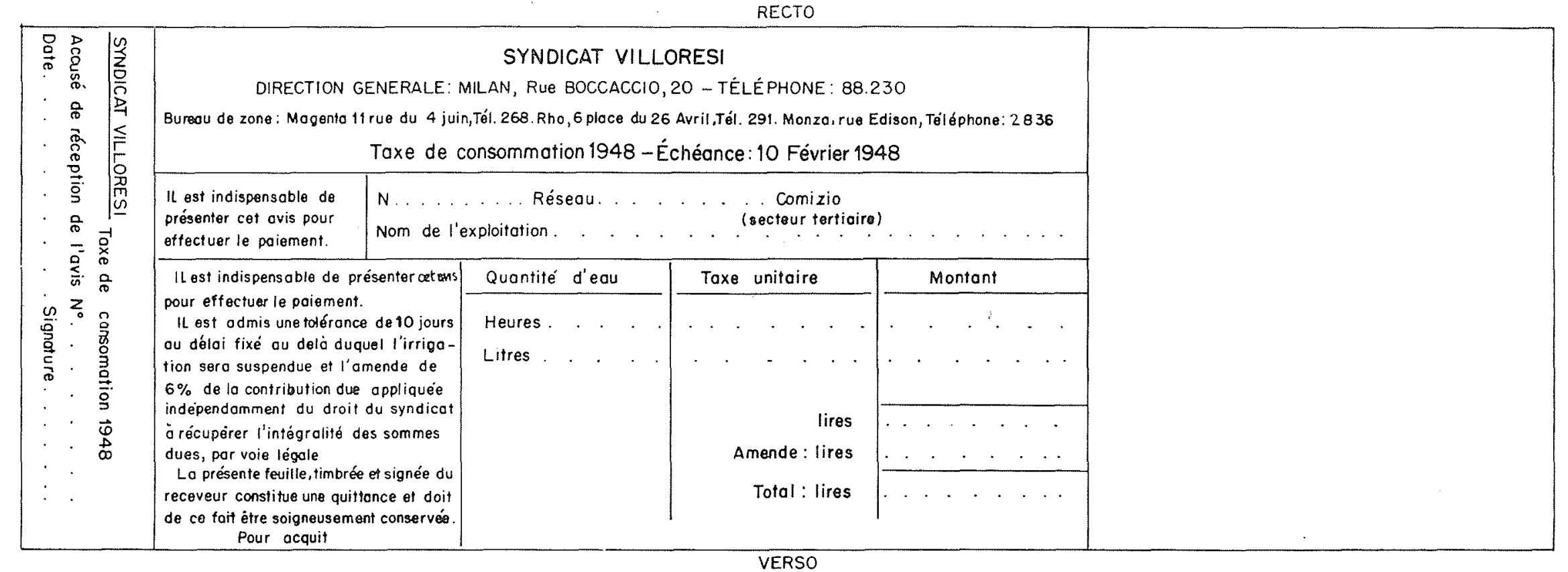

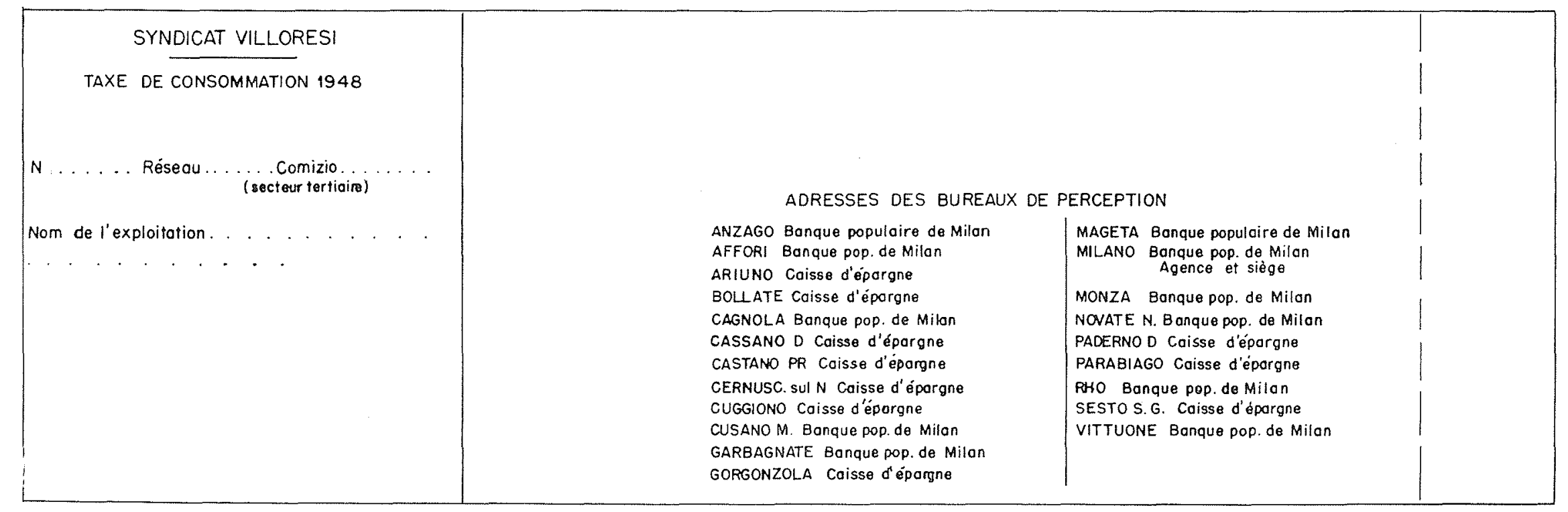




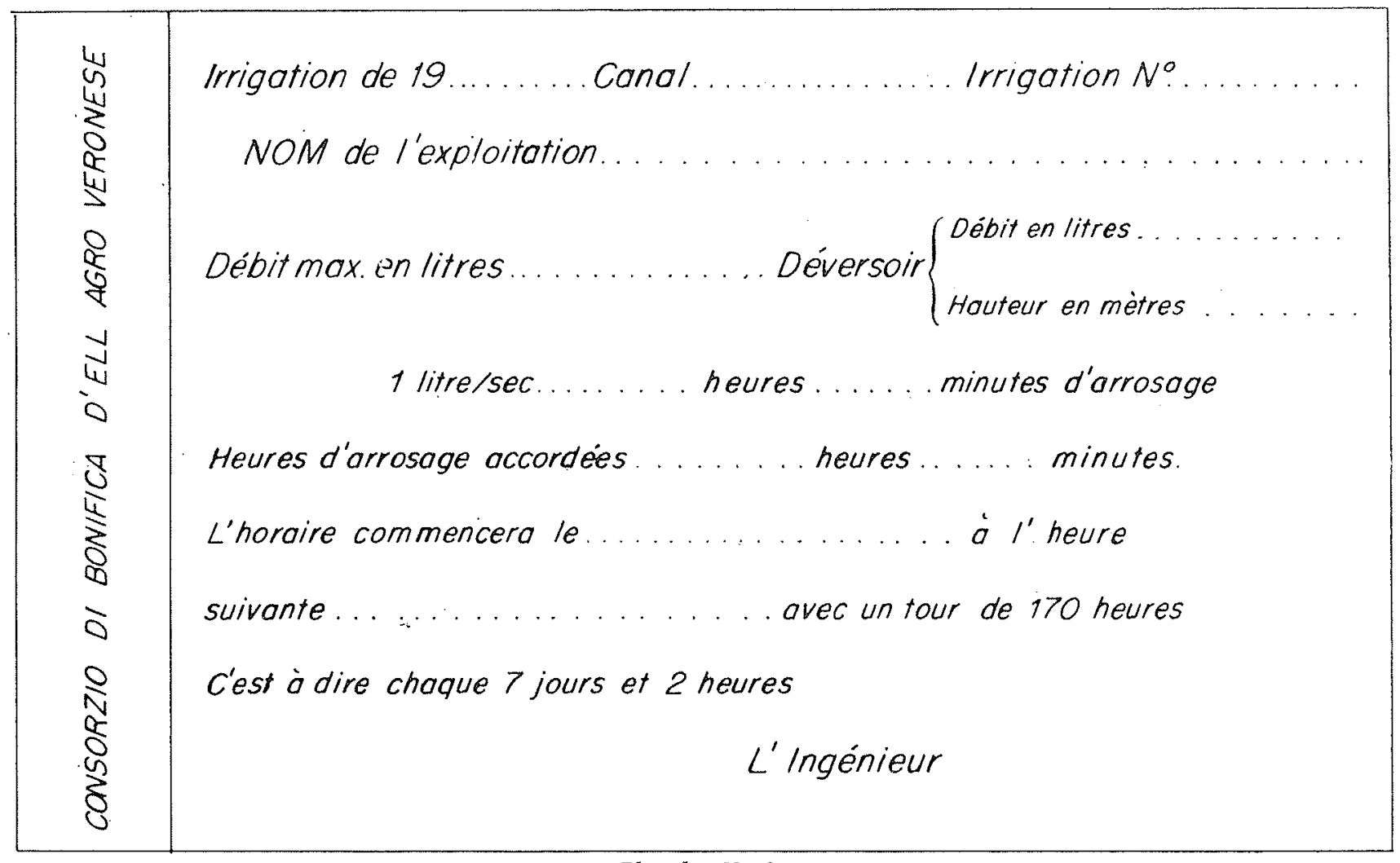

Planche $N^{\circ} 6$

\section{La distribution.}

Le principe reste le même. On attribue une main d'eau à un certain nombre d'usagers, de manière que chacun d'eux dispose de cette main d'eau une fois par semaine.

La main d'eau est en principe de 170 I./sec., car on cherche à se rapprocher de la formule:

1 heure d'arrosage $=11 \mathrm{l} / \mathrm{sec}$. continu

Ici la semaine est comptée à 170 heures, c'esta-dire deux heures de plus que la semaine effective, ceci afin qu'un usager donné n'arrose pas toujours à la même heure.

Ainsi, s'il a droit à deux heures d'arrosage, il aura :

la première semaine, le lundi, de $1 \mathrm{~h} .40$ à 3 h. 50 .

la deuxième semaine, le lundi, de $3 \mathrm{~h}, 40$ à 5 h. 50 .

la troisième semaine, le lundi, de 5 h. 40 à $7 \mathrm{~h} .50$, etc..

De cette manière, tous les arrosages de cet usager n'ont pas lieu de nuit.

Nous avons dit qu'en principe toutes les mains d'eau étaient de 170 1./sec. Suivant les travaux effectués dans les grands canaux du réseau, suivant les pertes en route, les disponibilités nettes d'eau varient chaque année. Ces variations, en somme, du débit total de principe du réseau, sont réparties sur chaque main d'eau qui ressortent à des valeurs voisines de 170 I./sec. : 160,25 - 168,40 (chiffres relevés). Dans certains cas où il est prouvé que l'usager a besoin de plus d'eau, la main d'eau atteint 2.00 et $2501 . / \mathrm{sec}$.

Le débit total est partagé dès le début de la saison et l'horaire peut être établi. Ce débit n'est pas influencé par les variations du débit de l'Adige, le débit de ce dernier étant toujours nettement supérieur aux prélèvements.

Pour l'établissement des redevances, l'eau est comptée à chacun comme si elle était fournie d'une manière continue.

Exemple relevé : main d'eau 160,25 1./sec.

1 I./sec. continu équivaut à 1 heure $3^{\prime} 39^{\prime}$ d'arrosage.

durée de l'arrosage hebdomadaire : $2 \mathrm{~h} .43^{\prime}$.

Cet arrosage correspond à un débit continu fictif de $2,57 \mathrm{l} . / \mathrm{sec}$. ; c'est sur ce dernier nom- 
bre qu'est établie la taxe qui s'élève à 2.500 lires au $1 . / \mathrm{sec}$.

Parmi les nombreux imprimés qu'utilise le consorzio, nous en reproduirons un (planche $\left.N^{\circ} 6\right)$.

Notons qu'en cas de pluie sur le réseau ei refus de l'eau par les usagers, la prise est avertie téléphoniquement pour qu'elle réduise son débit, non pas dans le but d'économiser de l'eau, mais pour éviter d'avoir trop d'eau à faire porter aux canaux de colatures parfois insuffisants.

\section{SYNDICAT DIRRIGATION D'EST-SESIA}

\section{Données généralles.}

Le Syndicat d'Irrigation d'Est-Sesia contrôle la distribution de l'eau sur un territoire de 190.000 hectares compris entre les cours de la Sesia, du Tessin et du Pô (planche $N^{*} 7$ ).

Le Syndicat s'est constitué en 1922 tout d'abord sous forme de société anonyme coopérative, puis, en 1927, il est légalement reconnu et l'engagement syndical est rendu obligatoire.

Les eaux utilisées par l'association sont celles de l'extrémité aval du canal Cavour dérivées du Pô à Chivasso et de la Doire Baltée à Saluggia et celles de la Sesia dérivées en plusieurs points de sa rive gauche.

Suivant les disponibilités des rivières qui l'alimentent, le réseau dispose d'un débit de 125 à $150 \mathrm{~m}^{3} / \mathrm{sec}$. Ce débit atteindra $200 \mathrm{~m}^{3} / \mathrm{sec}$. lorsque le canal Elena, dérivé du Tessin, sera définitivement construit.

Actuellement, 1.000 kilomètres de canaux principaux desservent la région, surveillés par une soixantaine de postes de garde reliés au bureau central du Syndicat à Novara par 400 kilomètres de lignes télégraphiques ou téléphoniques

Riz, maïs et prairies sont les cultures prédominantes de la région. Les 3 millions et demi de quintaux de riz récoltés en moyenne annuellement dans le réseau, constituent à eux seuls le $1 / 3$ de la production italienne. Le cheptel bovin évalué à 85.000 têtes et les 180 millions de litres de lait produits annuellement, placent cette région parmi les plus favorisées au point de vue de l'élevage et parmi les plus prospères de l'ltalie toute entière.

\section{Personmel.}

Une organisation aussi étendue exige un personnel qualifié ét nombreux.
M. I'Ingénieur Docteur Guido CANALINI assure la direction générale de l'Association, assisté de l'Ingénieur en Chef MARTELLI et de l'Inspecteur BUSSI.

Cinq bureaux se partagent, suivant leur spécialité, les différentes tâches du travail de gérance. Nous avons jugé utile de donner la composition de ces bureaux de manière à donner une idée de l'importance de l'organisme et du travail qu'il effectue.

\section{Un bureau hydrométrique.}

Ce bureau, relié téléphoniquement à tous les postes de garde et télégraphiquement aux principaux d'entre eux, est le véritable cœur du système.

11 centralise toutes les données hydrométriques, les exploite et diffuse tous les ordres de mancuvre qui en découlent, ceci grâce à un magnifique réseau de liaison.

Le central de ce réseau est équipé d'une façon moderne, comme on peut se rendre compte d'après la photographie de la planche No 9.

On y trouve en effet un central téléphonique relié à 76 postes téléphoniques, une machine à écrire télégraphique et un télégraphe. Le schéma de la planche 10 donne la disposition relative de ces différents points de liaison. Le local abrite aussi un enregistreur à distance du niveau du canal Cavour à l'entrée du réseau. On connaît ainsi à chaque instant le débit disponible en tête du réseau d'irrigation.

Le bureau hydrométrique est dirigé par un chef qui dispose d'un chef téléphoniste, d'un adjoint téléphoniste et d'un dessinateur.

Un bureau du Cadastre comprenant :

$$
\begin{aligned}
& \text { un chef du bureau, } \\
& \text { un agent technique, } \\
& \text { un dessinateur. }
\end{aligned}
$$

Ce bureau tient à jour l'état des propriétés. certaines attributions d'eau se faisant d'après la superficie.

\section{Bureau des projers et des travaux :}

un chef, quatre agents techniques, un dessinateur.

Le volume des travaux neufs est suffisant pour occuper tout le personnel énuméré. 


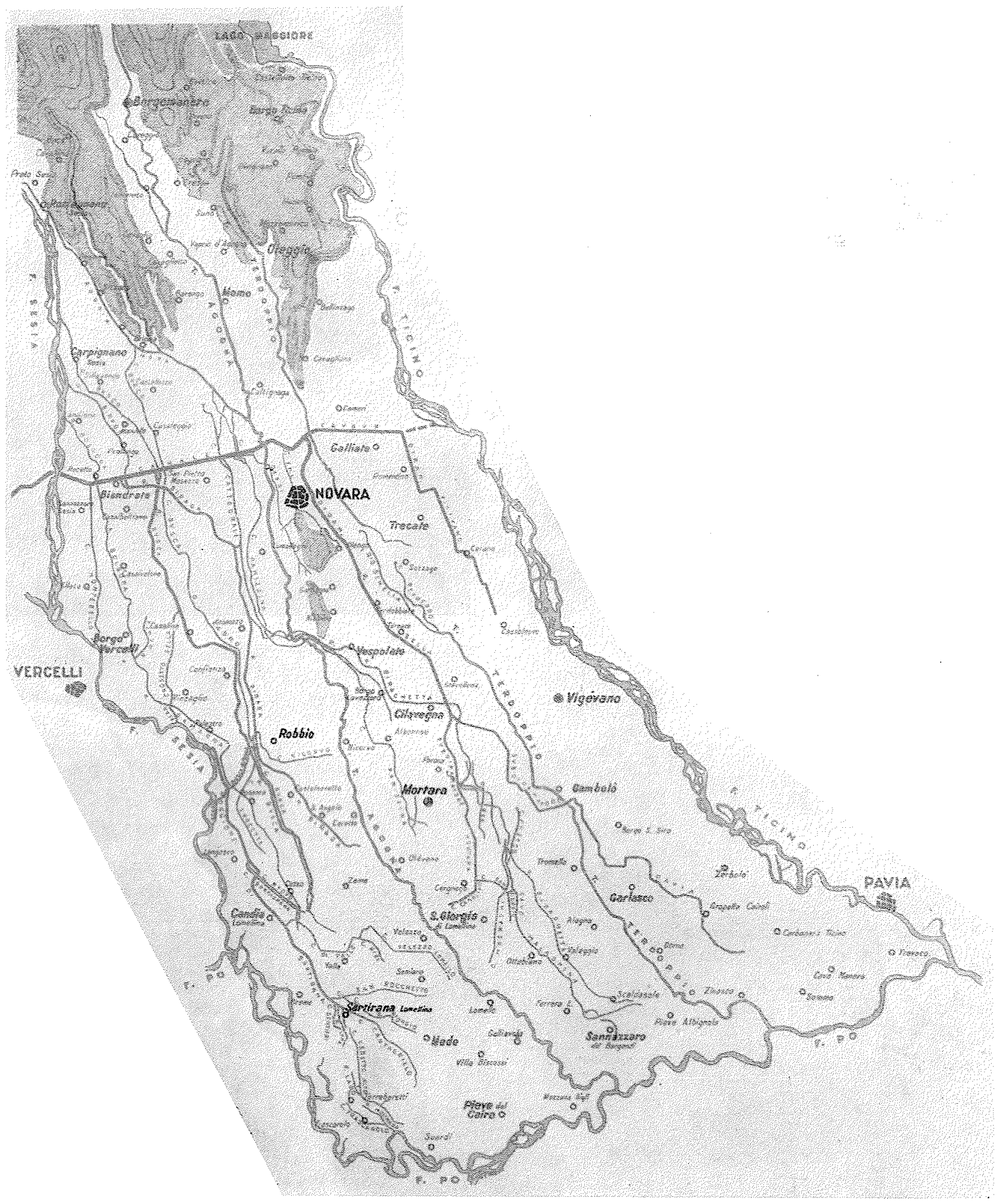

Planche $N^{\circ} 7$

Syndicat d'Irrigation d'Est Sesia

Carte générale du réseau 


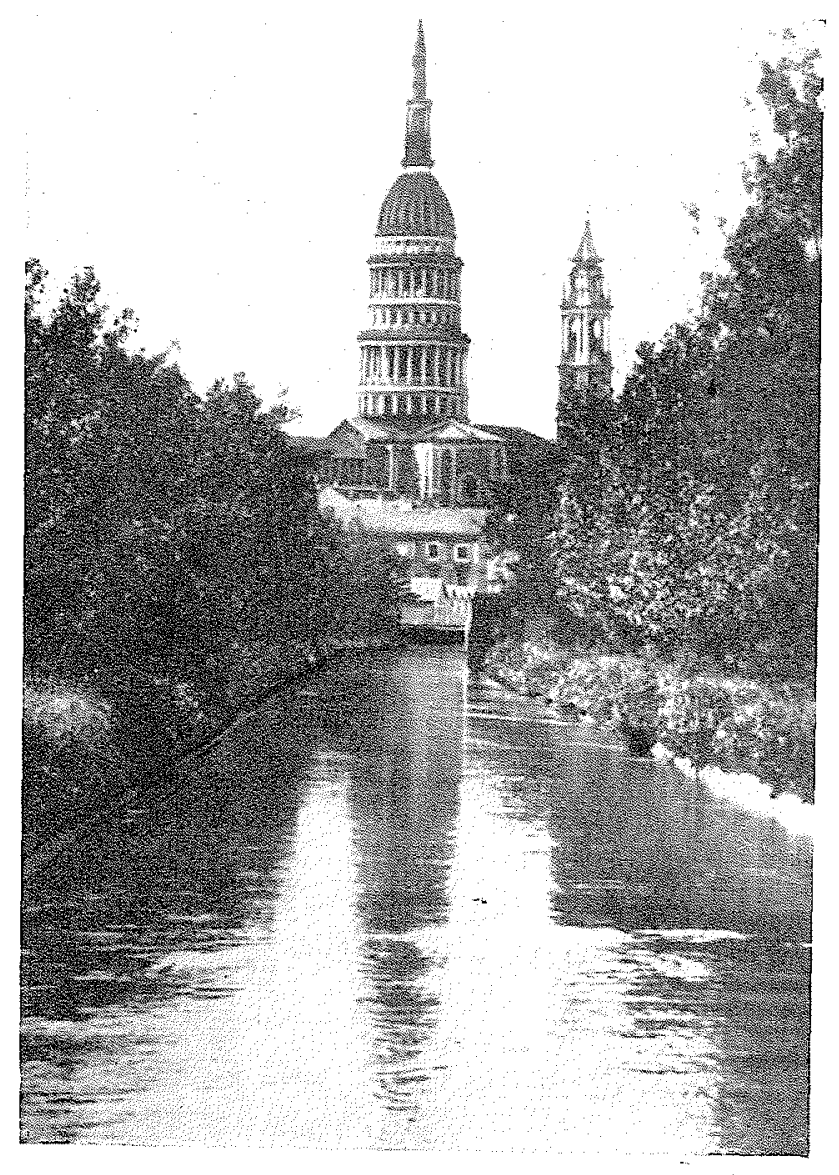

PLANCHE N ${ }^{\circ} 8$

$\longleftarrow$ Cavo Ricca

Subdiramatore Pavia
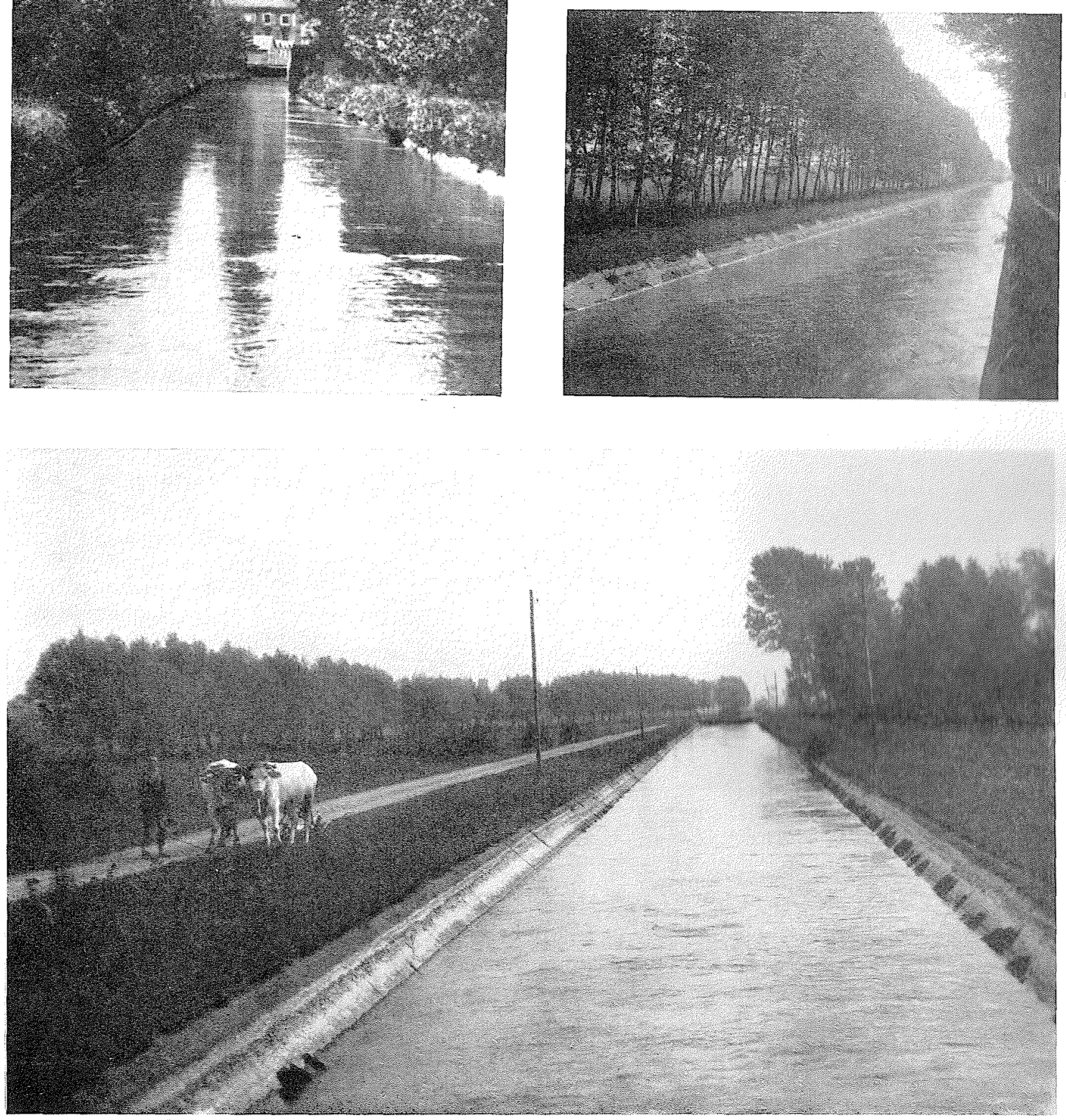

Cavo Montebello 


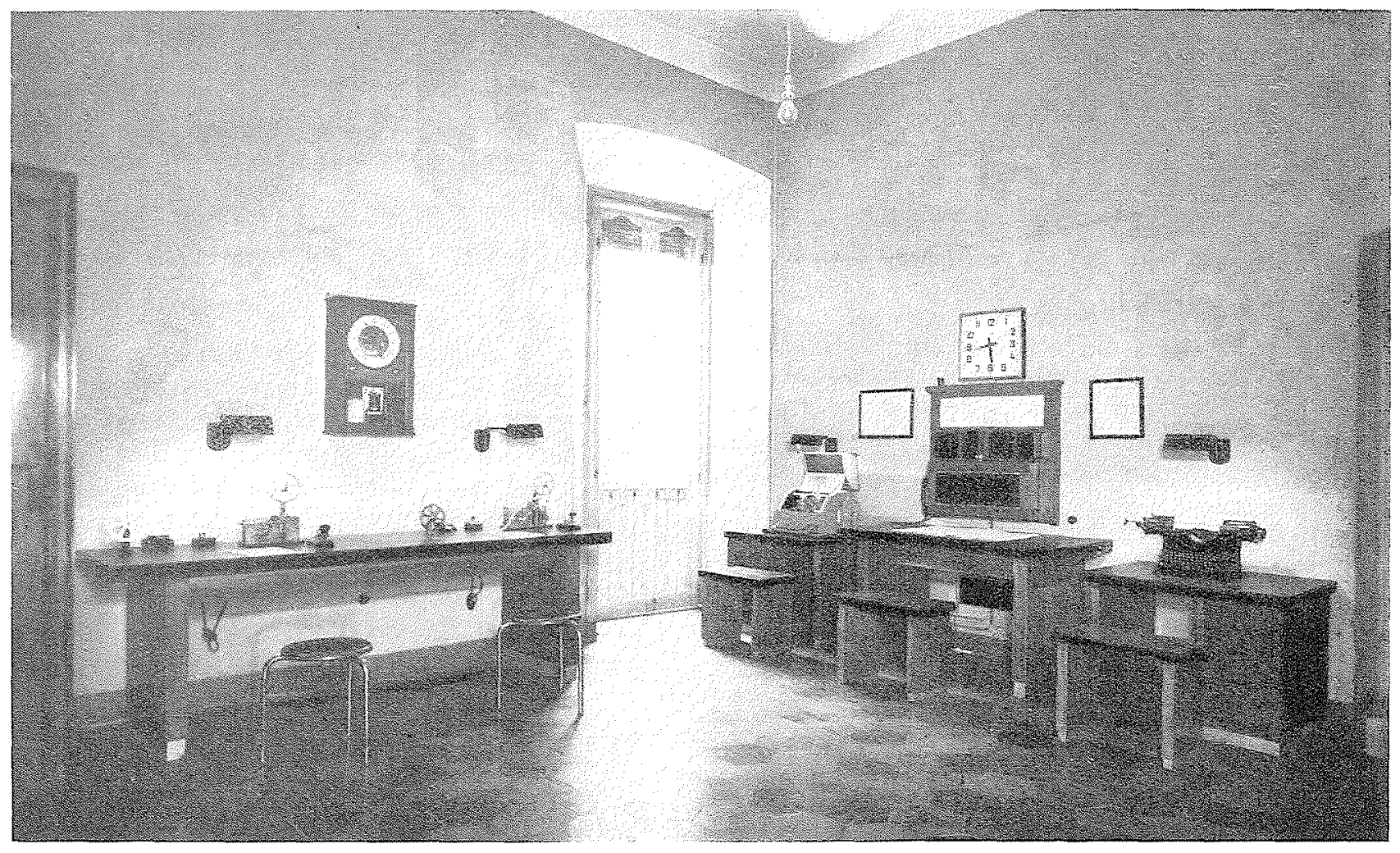

L'appareillage du bureau hydrométrique

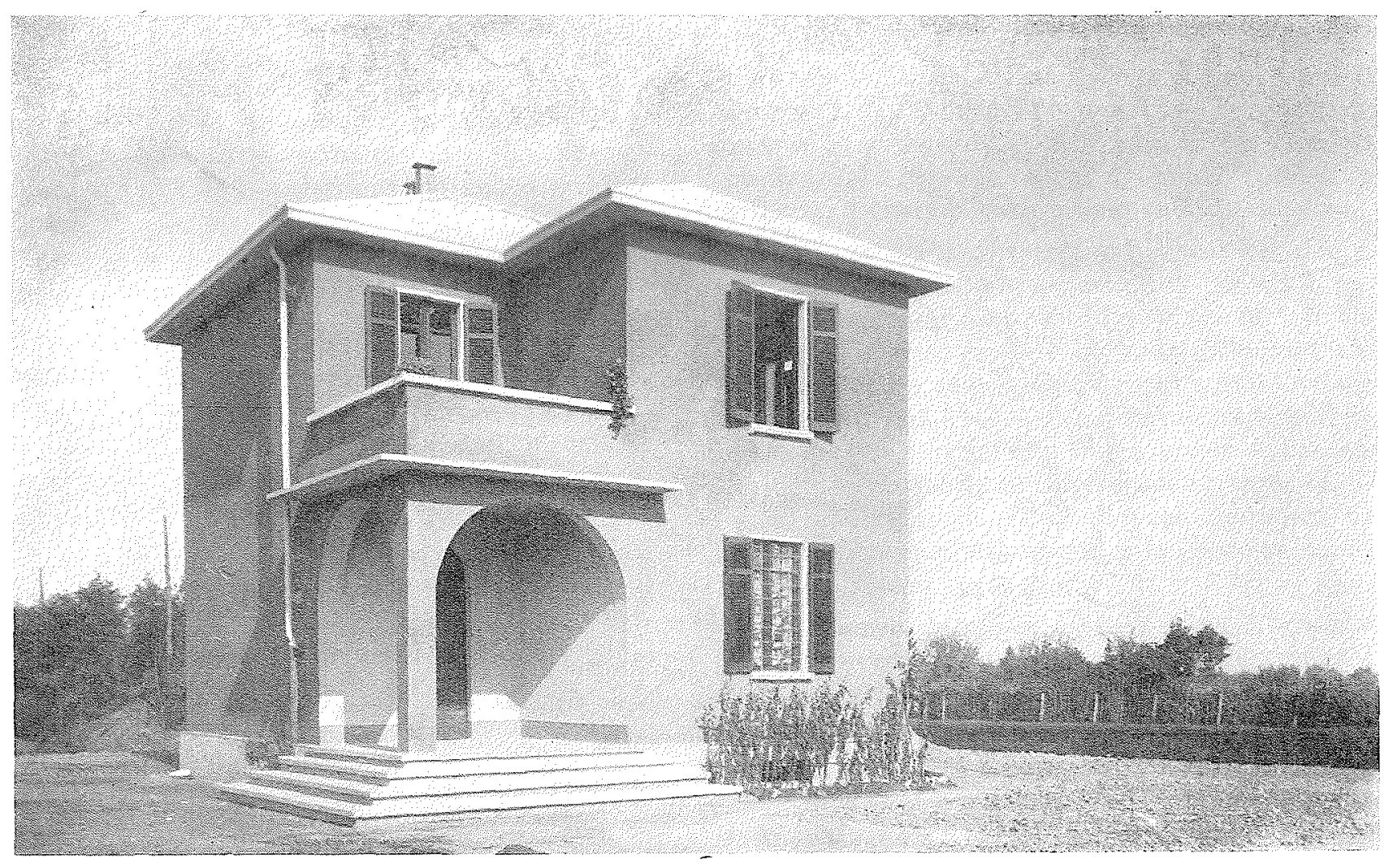

Type de maisonnette de garde des eaux

Planche $N^{\circ} 9$ 


\section{Bureau de l'Econome.}

Un ensemble aussi important exige un économat avec un économe assisté d'un aide-économe et d'un employé.

\section{Bureau de comprabilité.}

Enfin, un comptable et deux employés assurent le service de comptabilité de l'Association. qui doit déposer son bilan après chaque saison.

Le réseau est administrativement partagé en
6 zones: Novara, Mortara, Montebello, Robbio, Sartirana et Sella.

Chaque zone a son bureau de zone comprenant :

un chef de zone,

un agent technique,

$Z$ à 4 employés suivant l'importance de la zone et un certain nombre de gardiens, dont l'effectif pour l'ensemble du réseau atteint 64 .

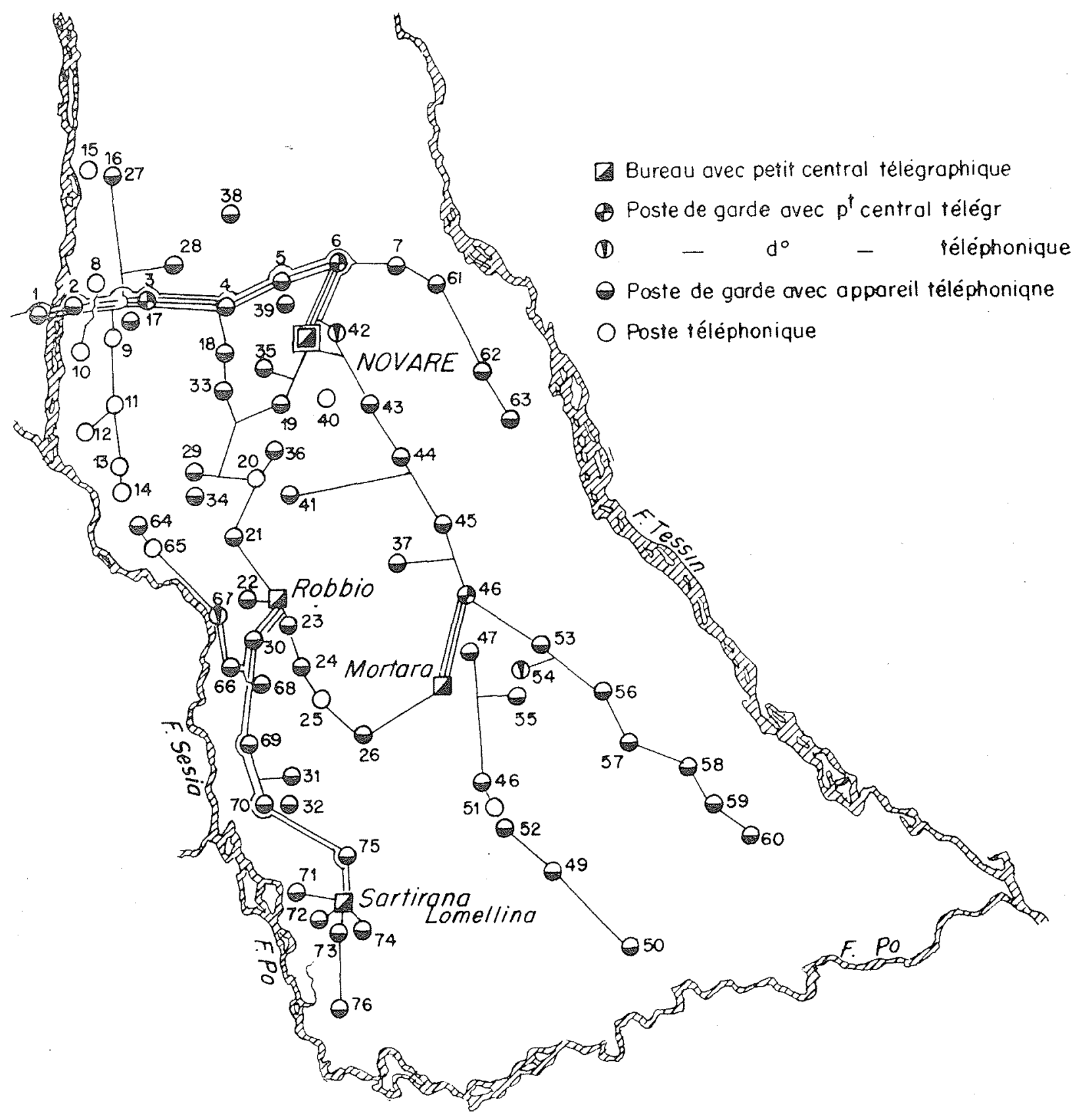

Planche No 10 
Ces derniers, logés dans les maisons de garde. sont reliés au moins téléphoniquement avec le siège central, à Novara.

\section{FONCTIONNEMENT DU SYNDICAT}

L'année est partagée en deux saisons:

une saison d'hiver, allant du l'r octobre au 28 février;

une saison d'été, allant du lor avril au 30 septembre,

le mois de mars étant réservé pour les travaux d'entretien des canaux alors à sec.

Les différentes parcelles du réseau sont dûment recensées par le bureau du Cadastre, qui tient à jour la liste des propriétaires, ces derniers faisant obligatoirement partie du Syndicat.

\section{Demandes.}

Avant chacume des deux saisons d'irrigation. chaque usager (ce n'est pas forcément le propriétaire, mais le locataire par exemple) présente une demande dont nous reproduisons l'imprimé à la planche II.

Pour la saison d'été, cette demande doit être remise en janvier.

Pour la saison d'hiver, l'imprimé doit être remis au bureau de zone au mois d'août. Pour éviter au bureau de confondre les deux demandes, les deux imprimés sont de couleur diffé. rente.

Le libellé même de la demande indique la manière dont l'eau est distribuée.

Nous voyons d'abord des attributions à prise mesurée.

Le débit attribué est dûment contrôlé par un déversoir (Bazin ou Rebbock) muni d'échelles limnimétriques et dont l'alimentation est réglable par une vanne levante manceuvrable à main.

Ce débit est soit continu (cas des rizières), soit intermittent (suivant un horaire établi au début de la saison comme pour le Syndicat Villoresi, avec une rotation de sept jours).

Dans le cas du débit continu, la demande précise la valeur de ce débit.

Dans le cas du débit discontinu, elle s'exprime en débit fictif continu Vibre.

Ensuite nous trouvons des attributions à prise

L'irrigation se fait sans détermination préalable d'horaire de rotation.
Le « comizio » comprend un certain nombre de "camperia » qui sont des unités territoriales de l'ordre de $150 \mathrm{~km}^{2}$. Chacune de ces «camperia » dispose d'une main d'eau qui est fixée à une valeur de principe une fois pour toutes (en général c'est le vieux droit d'eau qui est entériné).

Ce débit est affecté à tous les membres de la " camperia » suivant un ordre bien établi, mais non suivant un horaire. Le premier usager se sert; lorsque l'eau arrive au bout de son champ. il arrête l'arrosage et l'eau passe au deuxième, et ainsi de suite.

Si un usager ne veut pas arroser lorsque son tour arrive, son tour saute.

Suivant l'époque, suivant la facilité du terrain à absorber l'eau, selon qu'il est plus ou moins perméable et garni de végétation plus ou moins touffue, les séances d'arrosage durent plus ou moins longtemps et la rotation s'effectue donc en des temps variables.

\section{Manceuvres de distributhion.}

Ces demandes étant rassemblées, le bureau hydrométrique établit le calendrier des irrigations de la saison. L'opération est extrêmement simple. Il suffit, pour chaque canal, de faire la somme des demandes pour obtenir le débit à envoyer en tête du canal et celui qui est à affecter à chaque prise située le long du canal.

En poursuivant cette récapitulation des débits de chacun des canaux à partir des canaux d'extrémité jusqu'au canal principal, on obtient le débit à admettre en tête du canal principal. On définit ainsi le régime qui devrait s'établir dans le système, d'une manière uniforme tout au long de la saison d'irrigation. D'une année à l'autre, les demandes sont peu différentes et après quelques réductions de détail de ces dernières ce régime théorique correspond à la capacité maximum du réseau.

Le bureau de zone adresse alors à chaque garde un ordre de service (imprimé reproduit à la planche 12) qui, rappelant les caractéristiques des ouvrages placés sous le contrôle du garde, indique les débits que doivent régler chacun de ces ouvrages pour obtenir précisément ce régime théorique.

Mais les disponibilités du réseau sont souvent inférieures aux prévisions de ce programme. Le bureau hydrométrique connaît à tout moment le débit disponible par simple lecture de l'enregis- 


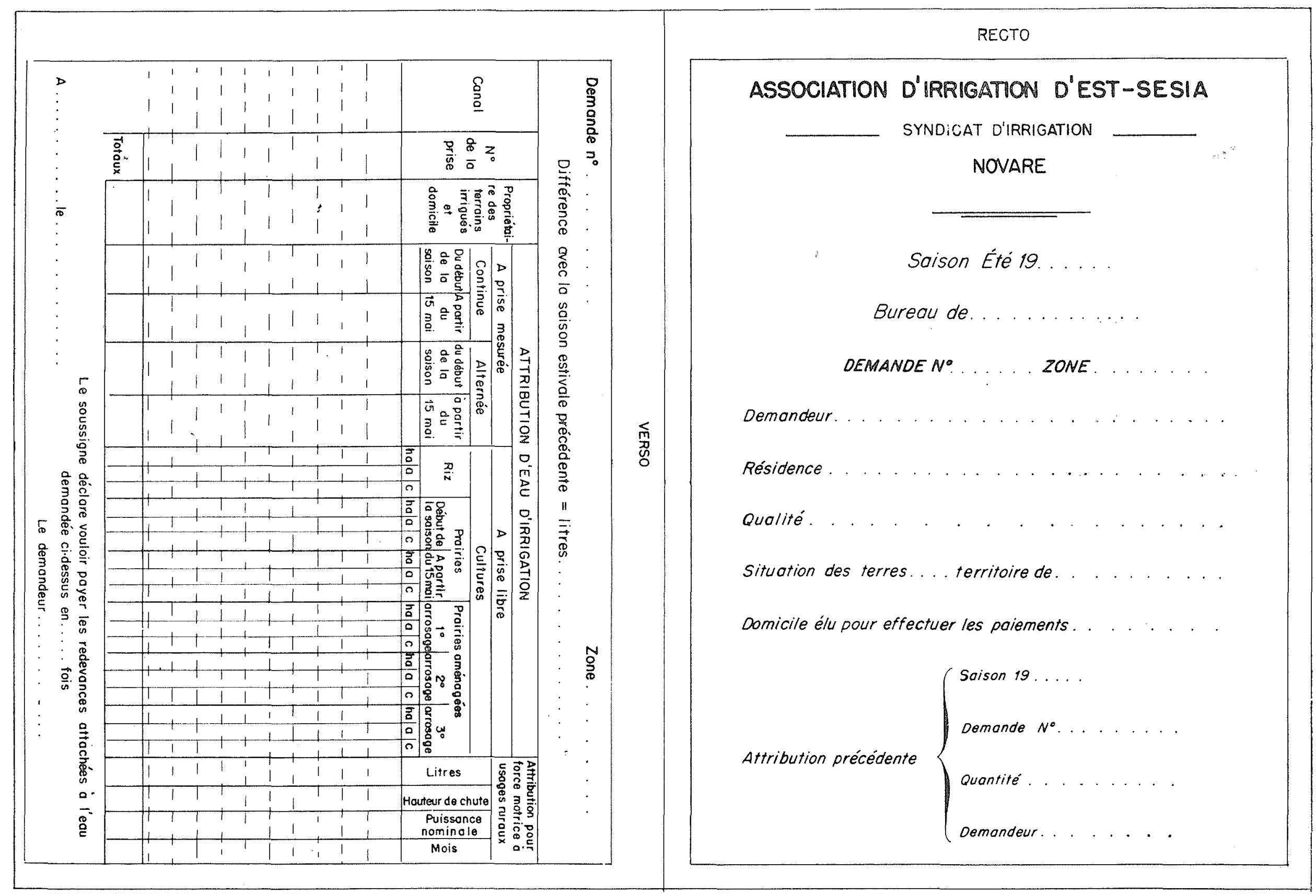




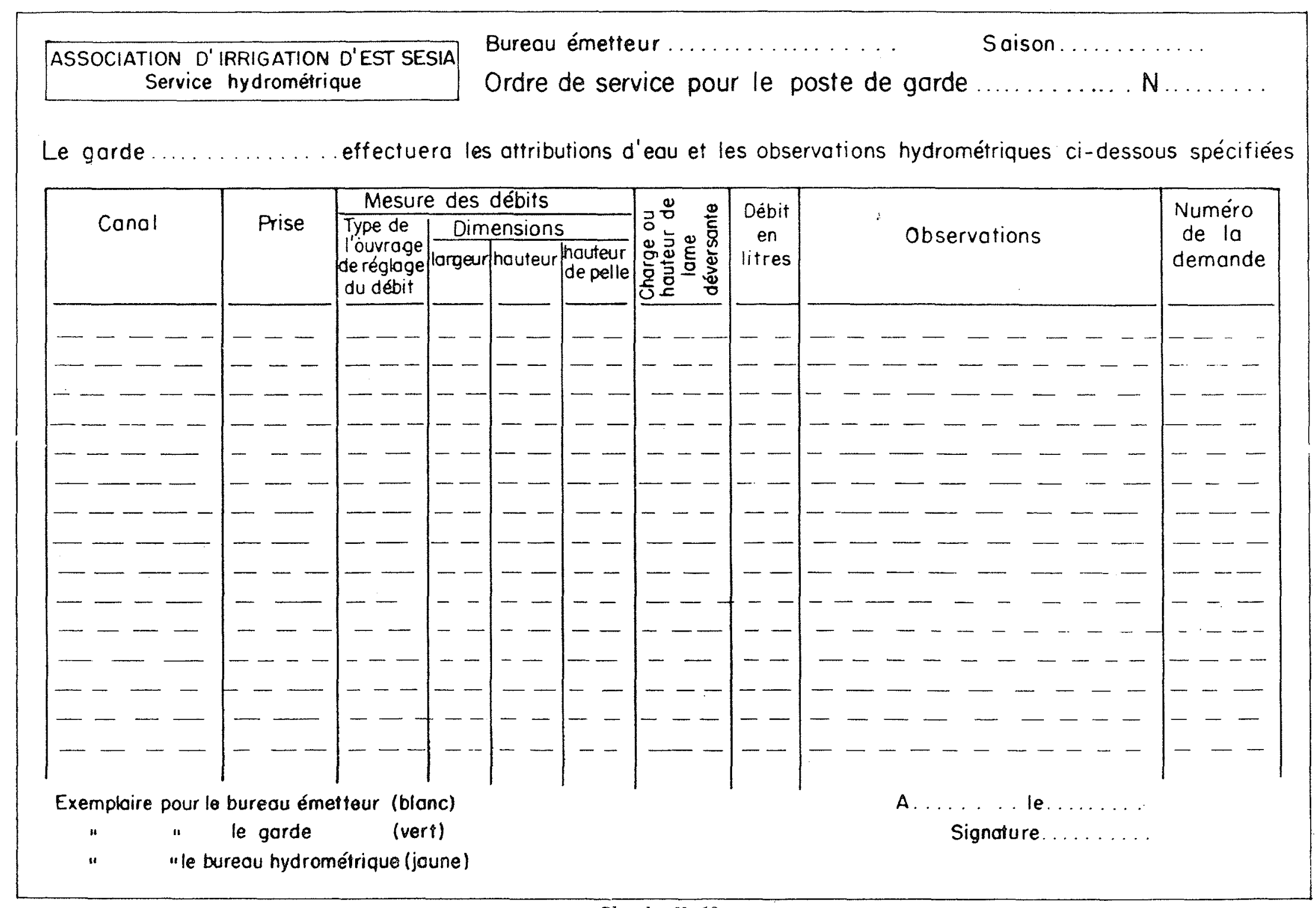

Planche $N^{\circ} 12$ 
treur qu'il possède dans la salle du central téléphonique. Chaque matin le bureau note le pourcentage du débit disponible par rapport au débit idéal. Grâce à l'un des moyens de communication, ce pourcentage est diffusé à tous les postes de garde qui, en revanche, signalent au bureau hydrométrique le pourcentage de réduction, dont leurs canaux sont affectés au moment où ils téléphionent.

Le bureau hydrométrique enregistre ainsi les débits réels des canaux, et le garde est averti que lorsque la variation annoncée en tête du réseau sera arrivée à la hauteur de son secteur, i) aura à régler le débit de ses ouvrages de manière à ce que ces attributions soient réduites dans la même proportion que celle qu'avait annoncée le siège central.

Pratiquement le garde sait, par expérience, qu'une variation de débit en tête du réseau lui parvient après un certain nombre d'heures. si bien qu'il effectue ses mancuvres à un moment bien déterminé par rapport à l'heure à laquelle est diffusé le pourcentage de réduction dont est affecté le canal principal.

Les liaisons téléphoniques ou télégraphiques constituent donc les outils de base de l'exploitation du réseau.

En fin de chaque saison, le bureau central donne la statistique des réductions effectivement exercées sur les prévisions.

Voici les réductions réalisées au cours de la saison 1947 sur trois des principales zones du réseau:

$$
\begin{array}{ll}
\text { Novara ... . . . } & 15,98 \% \\
\text { Mortara ... . . . . . . } & 16,68 \% \\
\text { Robbio . } 17,01 \%
\end{array}
$$

La proportion des réductions est donc sensiblement constante pour les différents secteurs du réseau.

\section{PRIX DE L'EAU}

Les redevances des usagers sont directement calculées à partir des demandes qu'ils ont faites. Avant la saison, le devis des dépenses est établi. ce qui permet de déterminer le prix de l'eau.

Suivant la manière dont les attributions sont faites, les prix varient. Nous en citerons quelques-uns extraits du tarif en cours pour la saison $1^{\text {cr }}$ mars 1948 - 28 février 1949.

\section{TARIF DE CONSOMMATION EN RÉSEAU NON RÉORGANISE Saison d'été}

\section{Irrigation à prise mesurếe :}

Arrosage continu

Arrosage intermittent

\section{Irrigation à prise libre:}

à partir
du début de saison ....
du 15 mai ..........
du début de saison .....
du 15 mai ..........

au $1 . / \mathrm{sec}$.

$\gg$

$》$

i)

\begin{tabular}{l|l} 
à 'ha & $7.500 »$ \\
$»$ & $5.000 \%$ \\
4.000 & $\gg$
\end{tabular}

1500 lires

$1.250 \gg$

$1.650 \gg$

1.375

\section{Saison d'hiver}

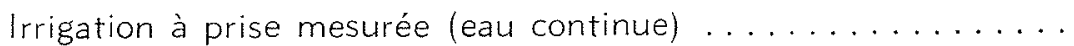

Irrigation à prise libre (consommation évaluée à $751 . / \mathrm{sec}$./ha) . . au $1 . / \mathrm{sec}$.

à I'ha

200

19.500 


\section{TARIF DE CONSOMMATION EN DISTRICT AUTONOME Saison d'été}

\section{lrugigation à prise mesurée :}

\begin{tabular}{|c|c|c|c|}
\hline Arrosage continu & 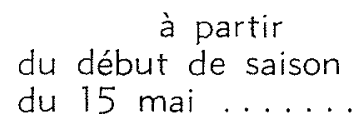 & au $1 . / \mathrm{sec}$. & $\begin{array}{l}1.300 \text { lires } \\
1.100\end{array}$ \\
\hline rrosage intermittent ... & $\begin{array}{l}\text { du début de saison } \\
\text { du } 15 \mathrm{mai} . . . . .\end{array}$ & $\begin{array}{l}\gg \\
\gg\end{array}$ & $\begin{array}{l}1.430 \\
1.210\end{array}$ \\
\hline
\end{tabular}

\section{Irrigation à prise libre:}

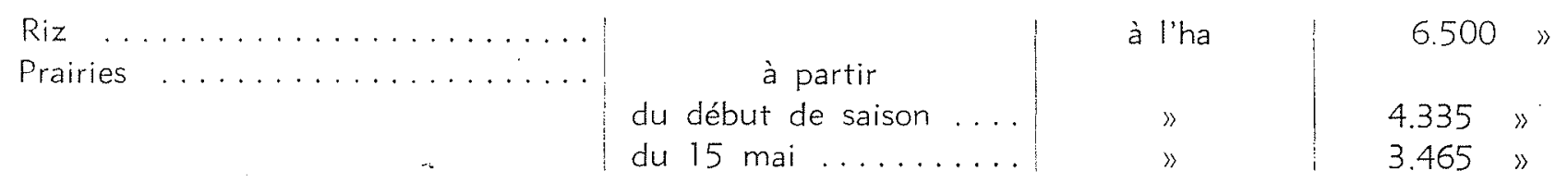

\section{Saison d'hiver}

Irrigation à prise mesurée (eau continue)

Irrigation à prise libre (évaluée à 75 I./sec./ha) au $1 . / \mathrm{sec}$.

à l'ha
$220 》$

$16.500 \gg$

\section{Contribution de nouvel ưhilisant :}

Pour la première année d'affiliation au Syndicat:

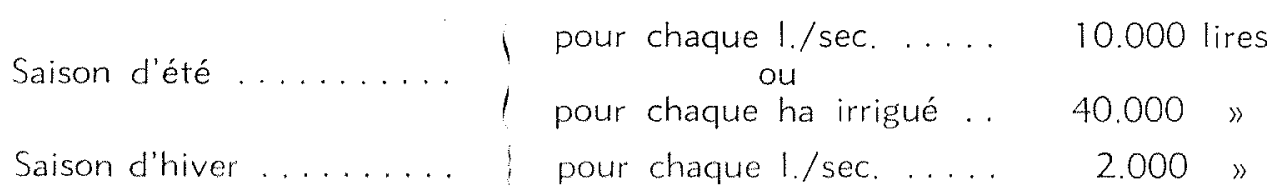

On remarquera que le litre/seconde de débit fictif est plus cher que le litre/seconde de débit réel. à cause des pertes d'eau et des manœuvres nécessitées par l'irrigation intermittente.

Les fortes consommations d'eau d'hiver s'expliquent par la curieuse pratique suivante: ce sont des prairies aménagées où le terrain a été spécialement préparé. La méthode est d'ailleurs très à l'honneur en Italie. Elle consiste à modeler le terrain de manière que la surface du sol se présente sous forme d'ondulations de 40 à $50 \mathrm{~m}$. de longueur d'onde. Les rigoles d'arrosage sont alors placées sur les crêtes et celles de drainage dans la ligne de thalweg. Les prairies ainsi aménagées reçoivent en hiver de l'eau en perma. nence, ce qui a comme principal avantage de réduire l'action du gel sur les herbes et de donner du fourrage extrêmement tôt. La formule a l'air d'être heureuse, puisque les agriculteurs consentent à payer 20.000 lires à l'hectare pour appliquer ce procédé. L'eau ruisselle donc le long des pentes des ondulations, se rassemble dans les creux et part à la colature, puis à des prairies situées en aval.

Remarquons aussi le tarif élevé de la cotisation d'inscription au Syndicat.

Pour donner une idée de la valeur intrinsèque de ces prix, notons que le cours du franc parmi les agents de change de Milan oscillait, en août 1948, autour de 1.55 lire.

\section{Bilan de l'Association.}

Grâce à la très grande amabilité du personnel de l'Association, nous sommes en mesure de donner un extrait du bilan de l'exercice 1947-48 tel qu'il fut déposé devant l'assemblée des délégués des membres de l'Association. 


\section{RECETTES \\ $1^{\circ}$ ENTRÉES EFFECTIVES}

1. Contributions syndicales:

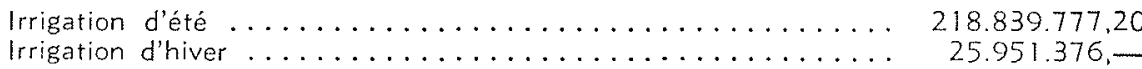

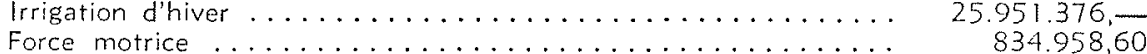

II. Intérêts, locations, redevances:

Intérêts ........................... 212.485,80

Loyers de moulins à eau, d'ateliers et de maisons . . . . . . . . . . 1.347.293,20

Entrées

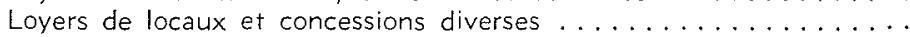

ordinaires

III. Revenus divers :

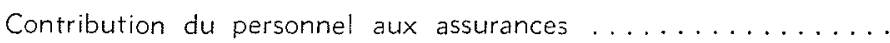

Remboursement de dépenses diverses $\ldots \ldots \ldots \ldots \ldots \ldots \ldots \ldots . . . \ldots$

Revenus divers

2.019

$6.488 .462,50$

330.507,

$254.013 .939,30$

Entrées extraordinaires

2.070 .131 .45

256.084 .070 .75

\section{FONDS DE ROULEMENT}

Recouvrement de fonds ................ 126.195.602,65

Divers ... . . . . . . . . . . . . . . . . . . . . $5.933 .588,30$

Versements syndicaux anticipés ............. 8.543.538,45

140.672 .729 .40

140.672 .729 .40

$396.756 .800,15$

DÉPENSES

$1^{\circ}$ DEPENSES EFFECTIVES

1. Redevances :

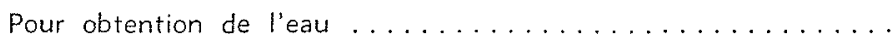

Pour des causes diverses.

$8.704 .059,25$

11. Travaux :

Entretien ordinaire des canaux, travaux extraordinaires ........

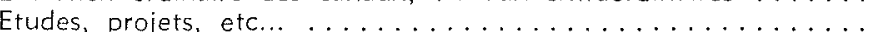

$108.126 .139,87$

$429.432,75$

111. Personnel :

Personne! de l'Etat (indemnités) $\ldots \ldots \ldots \ldots \ldots \ldots \ldots \ldots \ldots$

$669.934,18$

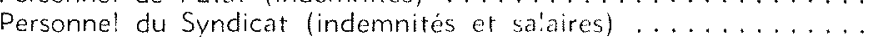

$77.869 .194,05$

IV. Locaux :

Location des locaux $\ldots \ldots \ldots$

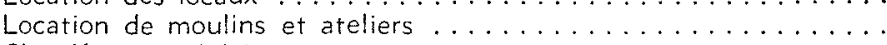

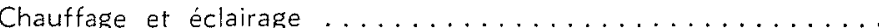

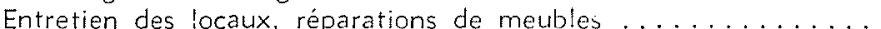

V. Impôts :

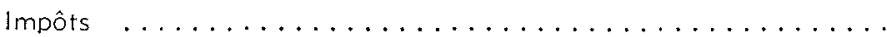

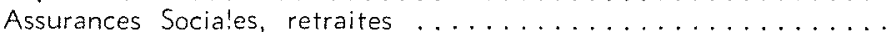

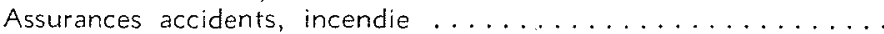

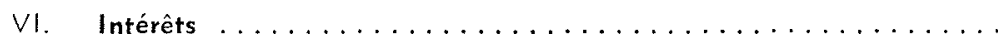

VII. Dépenses d'administration :

Indemnités remboursées aux membres des organes syndicaux ....

Secrétariat, timbres, téléphone, etc...............

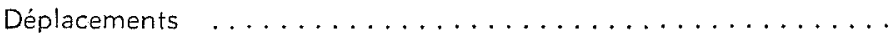

Dépenses extraordinaires

172.751,

$277.056,-$

146.646,

$3.160 .384,30$

$33.478 .584,65$

$845.148,15$

$4.574 .875,70$

525.217

$2.166 .960,20$

$2.900 .869,85$

$6.400 .808,-$

256.084 .070 .75 


\section{2" FONDS DE ROULEMENT}

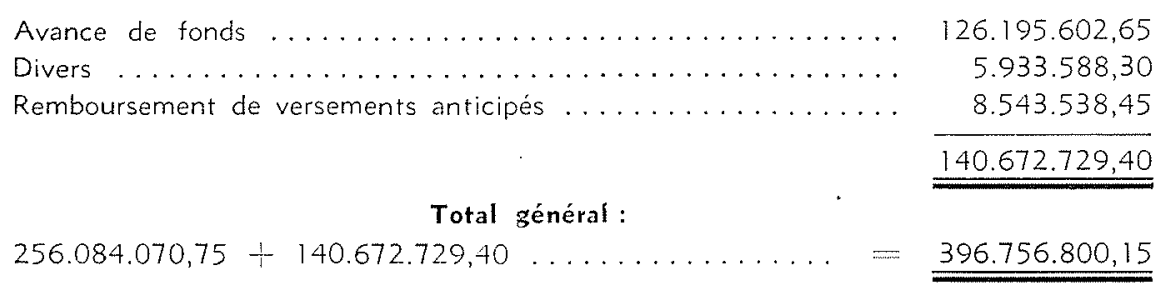

Constatons que sur environ 250 millions de lires de dépenses effectives, il faut attribuer

110 millions environ pour l'entretien des eaux et 80 millions environ pour le personnel d'exploitation

c'est-à-dire respectivement $44 \%$ et $32 \%$, et ceci pour une année où aucun travail neuf n'a été exécuté.

\section{CONCLUSIONS}

Nous nous excusons auprès du lecteur de la compilation fastidieuse de tous ces renseignements, mais nous avons pensé qu'il serait utile, pour l'ingénieur amené à s'occuper de l'exploitation d'un réseau, soit directement en en assurant le contrôle, soit indirectement en étudiant le projet d'irrigation d'un périmètre, de trouver des ordres de grandeur et des indications de nature à l'aider dans son travail.

Nous n'avons pas voulu donner les réseaux italiens en exemple, ni essayer l'ombre même d'une critique. mais simplement nous attacher à faire ressortir aussi fidèlement que possible ce que la pratique a elle-même sanctionné, le fait d'exister étant à notre avis une des preuves les plus probantes de la bonne marche d'un organisme.

Néanmoins, nous rappellerons que ces métho- des d'exploitation portent l'empreinte de certains caractères de l'Italie du Nord, caractères assez particuliers pour que ces méthodes ne soient pas directement applicables aux réseaux des autres pays. II convient de garder présent à la mémoire que les irrigations de la rive gauche du Pô intéressent des terrains excessivement perméables, que ces irrigations sont en grande partie pratiquées au fil de l'eau des rivières alpines, que ces rivières offrent des disponibilités peut-être non suffisantes eu égard aux besoins actuels, mais tout de même assez larges et que la pluviométrie de ces régions est suffisamment élevée pour qu'une déficience passagère de l'irrigation ne condamne pas irrémédiablement toute récolte.

Les trois réseaux visités ne nous permettent pas de dire qu'une méthode s'est vraiment imposée. Les règles de distribution ou de tarification varient d'un syndicat à l'autre et, comme toutes règles, ne valent que par l'application qu'on en fait.

Dans tous les cas, on remarquera que l'exploitation exige des moyens importants en personnel et en matériel, une machine administrative assez complexe malgré une distribution des plus simples et à peu près uniforme tout au long de la saison d'irrigation. 